\title{
Application Of a Genetic Algorithm To a Real World Nurse Rostering Problem Instance
}

\author{
Özgür Kelemci, A. Sima Uyar \\ Istanbul Technical University, Istanbul, Turkey \\ ozgurkelemci@yahoo.com,etaner@itu.edu.tr
}

Keywords: Genetic algorithms, personnel scheduling, nurse rostering, constraint handling, real world problems.

\begin{abstract}
The nurse rostering problem involves assigning shifts to qualified personnel using a given timetable under some hard and soft constraints. In this study, the nurse rostering problem instance of the Fatih Sultan Mehmet Hospital is solved using a standard genetic algorithm. Currently, the rosters are being prepared by a head nurse who performs this tedious task by hand. Due to the existence of many constraints, the resulting schedules are usually suboptimal. The aim in this study is to generate better schedules. This paper reports the results of the preliminary experiments for developing a good genetic algorithm for this problem.
\end{abstract}

\section{INTRODUCTION}

The nurse rostering problem (Burke et al., 2004) belongs to a group of hard real world problems more generally known as personnel scheduling. In the nurse rostering problem, the aim is to assign shifts to nurses who are qualified for the work they are assigned to do. Traditionally, schedules are prepared by hand. For automatic determination of the rosters, several approaches exist in literature such as evolutionary algorithms (Ahmad et al., 2000), simulated annealing (Brusco and Jacobs, 1995), tabu search (Burke and Soubeiga, 2003) and constraint programming (Cheng et al., 1997).

In this study, the nurse rostering problem of the Fatih Sultan Mehmet Hospital (FSMH) in Istanbul, Turkey is solved using genetic algorithms (GA). Currently, the head nurse in the hospital prepares the monthly schedules for the three departments by hand through "trial and error". Due to the existence of many hard and soft constraints, this procedure usually gives under-optimal schedules. The aim of the program developed in this study is to construct the monthly schedules automatically using a GA. The results of the experiments with a very basic implementation of a GA with standard features are very promising. Further research to improve the solution quality is being conducted.

\section{GENETIC ALGORITHMS}

GAs (Eiben and Smith, 2003) are a form of evolutionary algorithms which model mechanisms and ideas from Mendel's classical theory of genetics and the Darwinist evolutionary theory. In this study, a chromosome consists of the nurse schedules for all the nurses in the three departments of the hospital. Each day is represented as a gene which consists of two integer parts: the department id of the nurse and the shift type. There is a separate fitness calculation function for each constraint which has a weight assigned to it. The weighted sum of the fitness scores for the constraints gives the overall fitness score of the chromosome. The initial population is created randomly. The reproduction step consists of three stages: selection, crossover and mutation. In this study, tournament selection, uniform crossover and a random-resetting mutation is used. Trough a pure generational replacement scheme, the individuals to survive into the next generation are determined.

\section{THE NURSE ROSTERING PROBLEM}

In this study, the nurse rostering problem of the Fatih Sultan Mehmet Hospital (FSMH) in Istanbul, 
Turkey is solved. The actual data and the hard and soft constraints for generating the schedules are obtained from the hospital personnel and monthly schedules are created automatically. The output of the program determines the monthly schedules for all 11 nurses. In this hospital, if necessary, transfer of nurses between departments is possible.

There are four shift types. "Empty Shift", "Day Shift" and "Night Shift" (these shifts occurs during the weekdays), "Combined Shift" (covers the whole day and occurs only on the weekend days). There are three departments: "Urology" and "Neurology" are small departments as opposed to the "Internal Medicine" department. The hard ("H") and soft ("S") constraints are given below. The settings for each constraint are determined based on historical data of previous schedules obtained from the hospital and on requirements stated by the head nurse forming the schedules by hand. The schedules are prepared monthly but for $\mathrm{H} 1, \mathrm{H} 2$ and $\mathrm{H} 3$, last two days of the previous month are also considered.

Weekend Shift Distribution Constraint (H1): Only one combined shift may be assigned for consecutive weeks.

Off-Day Constraint (H2): If a nurse works a night or a combined shift, an empty shift is assigned for the next day.

Days Between Shifts Constraint (H3): There should be two days between two consecutive night or combined shifts for each nurse.

Night and Combined Shift Staff Distribution Constraint (H4): Exactly two nurses should be assigned for each night and combined shift.

Repetition on Weekday Constraint (H5): At most two night shifts can be assigned for the same weekday in a month.

Weekend Shift Constraint (H6): Combined shifts should not be assigned for the same day.

Lower Limit for Day Shift Distribution Constraint (H7): At least 4 nurses for the internal medicine and 1 nurse each for the smaller departments should be assigned for each day shift.

Working Days for the 15-day Annual Leave Constraint (H8): Night or combined shifts may be assigned to the first Monday after the 15-days annual leave and to the first Thursday before.

Off-Days for Annual Leave constraint (H9): Combined shifts should not be assigned for the first weekend after and the first weekend before the annual leave.

Weekend Shift Distribution Constraint (S1): At most 3 and at least 1 night and combined shifts can be assigned to each nurse.
In Department Constraint (S2): Nurses of the small departments should be assigned to their departments for the day shifts. This constraint is corrected by exchanging assignments with a nurse in another department assigned to the same shift.

Night Shift Distribution Constraint (S3): Nurses from the same small departments should not work in the same night and combined shifts.

Weekend Pair Constraint (S4): Different weekend-shift pairs should be assigned for consecutive months.

Night Shift on Weekdays Constraint (S5): At most 5 and at least 1 night shifts can be assigned.

Working Days for the 5-day Annual Leave Constraint (S6): Night or combined shifts may be assigned to the first Monday and to the first Thursday before the 5-days annual leave.

As stated before, the fitness of an individual is the weighted sum of its penalty scores. In some of the experiments, this value is used as it is and in some, it is normalized. The objective is to minimize the fitness value. The constraints have weights associated with them. In the experiments section, some of the experiments are conducted with different fixed penalty weights for hard and soft constraints. In the remaining experiments, adaptive weights are used to change the weights assigned to soft constraints in time. A change mechanism is chosen such that in the beginning, the weights are lower and they increase over time and the adaptive weight function assigns a weight value between zero and one as can be seen in Eq. 1 where $\mathrm{E}$ is the adaptive weight constant.

$$
\text { weight }=1-\mathrm{e}^{-(\text {Number of Current Generation/E) }}
$$

\section{EXPERIMENTS}

The genetic algorithm setup used in all of the experiment cases is given in Table-1.

18 different test scenarios are listed in Table- 2 . In the table, the first column gives the test id and the remaining columns either show whether the corresponding operator/method is used in the test case or not or give the corresponding value of a parameter chosen for the specific test case. Case results are listed in Table-3. $\alpha$ shows the percentage of success over 50 runs. Success is defined as obtaining a solution which is the global optimum, i.e. has a total penalty score (fitness) of $0 . \beta$ shows the percentage of solutions over 50 runs which do not violate any hard constraints but may or may not violate some soft constraints. The numbers in the 
remaining columns show the number of times the corresponding constraint was violated over 50 runs.

Table 1: Genetic Algorithm Setup

\begin{tabular}{|c|c|}
\hline GA Type & Generational \\
\hline Chromosome Length (L) & $11 *$ No of days in month \\
\hline Population Size & 330 \\
\hline Max No of Generations & 5000 \\
\hline Crossover & Uniform, rate $=1$ \\
\hline Mutation & Traditional mutation, rate $=1 / \mathrm{L}$ \\
\hline Mate Selection & Tournament selection, $\mathrm{k}=8$ \\
\hline Hill Climbing Selection & Tournament betw. 4 constraints \\
\hline Run Count & 50 \\
\hline
\end{tabular}

Results show that $\mathrm{C} 17$ is the best test scenario in terms of success ratio to get the best fitness value in successful runs. In this test case, all operators and mechanisms, i.e. adaptive soft constraint weights, normalization of penalty scores, repair through hill climbing and increased weight for the $3^{\text {rd }}$ hard constraint (H3) are applied. There is only one difference between case 7 and case 17, which is the weight of the hard constraint H3. It was observed in the initial testing stages that, considering the last two days of the previous month causes more penalty points for constraint $\mathrm{H} 3$ and is hard to resolve, so the weight of $\mathrm{H} 3$ is increased (it is set to 10 as opposed to 1 for all other hard constraints) in cases $\mathrm{C} 17$ and C18 to remedy this problem. However, this increases the penalty score of $\mathrm{H} 4$ and $\mathrm{H} 2$ in $\mathrm{C} 17$ and increases the penalty score of some of the soft constraints in C18. C18 is the best test scenario based on $\beta$.

It can be seen from the results that using the repair method which basically is a hill climbing operator, increases the success ratio. The normalization method improves the success ratio. The adaptive weight method increases the weight of the soft constraints exponentially, so the penalty points of soft constraints decrease, but this affects hard constraints due to the fact that the relative effect of the weights of the hard constraints on the overall fitness also decreases.

\section{CONCLUSION AND FUTURE WORK}

A real world instance of the nurse rostering problem is solved using mostly a standard GA. Actual data and hard and soft constraints have been obtained from a hospital (FSMH in Istanbul, Turkey) where currently the head nurse of the hospital is preparing the schedules by hand. The effect of two constraint handling methods, a repair technique, normalization of fitness values and parameter settings for these are explored in this study. As a result of the experiments, it is seen that normalization of the penalty scores, repairing of constraint violations and using adaptive weights for the constraints are all useful to obtain good results. During the experiments trying to satisfy the hard constraint $(\mathrm{H} 3)$ which seemed to be a problematic constraint, it is seen that it could also be useful to give different weights to different constraints. It would be worthwhile to explore this in the future.

Since this study aims to solve a real world problem, it is expected that it answers all the requirements of the hospital personnel for which it is designed. This study presents the results of preliminary experiments. Even though a very standard GA is used, the results are quite promising. After further experiments with fine tuning the current approach, experiments with more sophisticated GAs will be performed. Further work is currently being conducted to address these issues.

\section{REFERENCES}

Burke, E. K., de Causmaecker, P., vanden Berghe, G.,van Landeghem, H., 2004. "The State of the Art of Nurse Rostering". 7:411-499. Kluwer.

Ahmad, J., Yamamoto, M., Ohuchi A., 2000. "Evolutionary Algorithms for Nurse Scheduling Problem", Proc. of IEEE Congress on Evolutionary Computation, 196-203.

Brusco, M. J., Jacobs, L. W., 1995. "Cost Analysis of Alternative Formulations for Personnel Scheduling in Continuously Operating Organizations". European J. of Operational Research, 86:249-261.Elsevier.

Burke, E., Soubeiga, E., 2003. "Scheduling Nurses using a Tabu-Search Hyperheuristic", Proc. of MISTA, 197218.

Cheng, B.M.W., Lee, J.H.M., Wu, J.C.K.,1997. “A Nurse Rostering System using Constraint Programming and Redundant Modelling”, IEEE Trans. On Information Technology in Biomedicie, 1(1):44-54.

Eiben, A. E., Smith, J. E., 2003. Introduction to Evolutionary Computing, Springer. 


\begin{tabular}{|c|c|c|c|c|c|c|c|c|}
\hline \multicolumn{9}{|c|}{ Table-2. Description of the test cases } \\
\hline Cases & $\begin{array}{c}\text { Adap } \\
\text { tive }\end{array}$ & $\begin{array}{c}\text { Norma } \\
\text { lization }\end{array}$ & Repair & $\begin{array}{c}\text { Exch } \\
\text { ange }\end{array}$ & $\begin{array}{c}\text { Soft/Hard } \\
\text { Weight }\end{array}$ & $\begin{array}{c}\text { Constraint S6 } \\
\text { (Low/High Limit) }\end{array}$ & $\begin{array}{c}\text { H3 } \\
\text { Weight }\end{array}$ & E \\
\hline C1 & No & No & No & No & $0.1 / 1$ & $1 / 5$ & 1 & - \\
\hline C2 & No & No & Yes & No & $0.1 / 1$ & $1 / 5$ & 1 & - \\
\hline C3 & No & Yes & Yes & No & $0.1 / 1$ & $1 / 5$ & 1 & - \\
\hline C4 & Yes & No & Yes & No & $(0.02-1) / 1$ & $1 / 5$ & 1 & 500 \\
\hline C5 & Yes & Yes & Yes & No & $(0.02-1) / 1$ & $1 / 5$ & 1 & 500 \\
\hline C6 & No & No & Yes & Yes & $0.1 / 1$ & $1 / 5$ & 1 & - \\
\hline C7 & Yes & Yes & Yes & Yes & $(0.02-1) / 1$ & $1 / 5$ & 1 & 500 \\
\hline C8 & No & Yes & Yes & Yes & $0.1 / 1$ & $1 / 5$ & 1 & - \\
\hline C9 & Yes & Yes & Yes & Yes & $(0.02-1) / 1$ & $1 / 5$ & 1 & 100 \\
\hline C10 & No & Yes & Yes & Yes & $0.1 / 1$ & $1 / 5$ & 1 & - \\
\hline C11 & Yes & Yes & Yes & Yes & $(0.02-1) / 1$ & $2 / 3$ & 1 & 500 \\
\hline C12 & No & Yes & Yes & Yes & $0.1 / 1$ & $2 / 3$ & 1 & - \\
\hline C13 & Yes & Yes & Yes & Yes & $(0.02-1) / 1$ & $2 / 3$ & 1 & 500 \\
\hline C14 & No & Yes & Yes & Yes & $0.1 / 1$ & $2 / 3$ & 1 & - \\
\hline C15 & No & Yes & Yes & Yes & $0.1 / 1$ & $2 / 3$ & 1 & - \\
\hline C16 & No & Yes & Yes & Yes & $0.1 / 1$ & $1 / 5$ & 1 & - \\
\hline C17 & Yes & Yes & Yes & Yes & $(0.02-1) / 1$ & $1 / 5$ & 10 & 500 \\
\hline C18 & No & Yes & Yes & Yes & $0.1 / 1$ & $1 / 5$ & 10 & - \\
\hline
\end{tabular}

\begin{tabular}{|c|c|c|c|c|c|c|c|c|c|c|c|c|c|c|c|c|c|}
\hline \multicolumn{18}{|c|}{ Table-3. Results of the tests for all test cases } \\
\hline & H1 & $\mathrm{H} 2$ & $\mathrm{H} 3$ & $\mathrm{H} 4$ & H5 & H6 & $\mathrm{H} 7$ & $\mathrm{H} 8$ & H9 & S1 & S2 & S3 & S4 & S5 & S6 & $\alpha$ & $\beta$ \\
\hline $\mathrm{C} 1$ & 8 & 1 & 1 & 36 & - & 1 & - & - & - & 17 & 45 & 29 & 48 & 3 & - & 0.00 & 0.20 \\
\hline $\mathrm{C} 2$ & - & - & - & 1 & - & - & - & - & - & 4 & 20 & 1 & 24 & - & - & 0.26 & 0.98 \\
\hline C3 & - & - & 15 & - & - & - & - & - & - & 3 & 11 & 5 & 3 & - & - & 0.48 & 0.70 \\
\hline $\mathrm{C} 4$ & - & - & - & 27 & - & 2 & - & - & - & 10 & 45 & 18 & 37 & 2 & - & 0.02 & 0.36 \\
\hline $\mathrm{C} 5$ & - & - & 26 & - & - & - & - & - & - & - & - & - & - & - & - & 0.48 & 0.48 \\
\hline C6 & - & - & - & - & - & - & - & - & - & 1 & 4 & 4 & 21 & - & - & 0.46 & 1.00 \\
\hline C7 & - & - & 18 & - & - & - & - & - & - & - & - & - & - & - & - & 0.64 & 0.64 \\
\hline C8 & - & - & 9 & - & - & - & - & - & - & 3 & 3 & 3 & - & - & - & 0.74 & 0.82 \\
\hline C9 & - & 2 & 22 & 2 & - & - & - & - & - & - & - & - & - & - & - & 0.56 & 0.56 \\
\hline C10 & - & - & 4 & - & - & - & - & - & - & 2 & 2 & 3 & 12 & - & - & 0.58 & 0.92 \\
\hline C11 & - & 1 & 34 & 3 & - & - & - & - & - & - & - & - & - & - & - & 0.32 & 0.32 \\
\hline $\mathrm{C} 12$ & - & - & 10 & - & - & - & - & - & - & 1 & 5 & 3 & 3 & 15 & - & 0.42 & 0.80 \\
\hline C13 & - & 4 & 38 & 4 & - & - & - & - & - & - & - & - & - & - & - & 0.24 & 0.24 \\
\hline C14 & - & - & 3 & - & - & - & - & - & - & 4 & 4 & 2 & 13 & 6 & - & 0.52 & 0.94 \\
\hline $\mathrm{C} 15$ & - & - & 27 & 1 & - & - & - & - & - & 1 & - & - & - & 1 & - & 0.42 & 0.44 \\
\hline C16 & - & - & 23 & - & - & - & - & - & - & - & - & - & - & - & - & 0.54 & 0.54 \\
\hline C17 & - & 2 & - & 18 & - & - & - & - & - & - & - & - & - & - & - & 0.78 & 0.78 \\
\hline C18 & - & - & - & - & - & - & - & - & - & 4 & 3 & 1 & 12 & - & - & 0.70 & 1.00 \\
\hline
\end{tabular}

